\title{
PRIMARY IMMUNE SYSTEM RESPONDERS TO NUCLEUS PULPOSUS CELLS: EVIDENCE FOR IMMUNE RESPONSE IN DISC HERNIATION
}

Kunihiko Murai ${ }^{1 *}$, Daisuke Sakai², ${ }^{3}$, Yoshihiko Nakamura ${ }^{3}$, Tomoko Nakai $^{3}$, Takashi Igarashi ${ }^{1}$, Norimasa Seo ${ }^{1}$, Takashi Murakami ${ }^{4}$, Eiji Kobayashi ${ }^{4}$, and Joji Mochida ${ }^{2,3}$

${ }^{1}$ Department of Anesthesiology and Intensive Care Medicine, Jichi Medical University, 3311-1 Yakushiji, Shimotsuke, Tochigi, 329-0498, Japan

${ }^{2}$ Department of Orthopaedic Surgery, Surgical Science, Tokai University School of Medicine, 143 Shimokasuya, Isehara, Kanagawa, 259-1193, Japan

${ }^{3}$ Research Center for Regenerative Medicine, Tokai University School of Medicine, 143 Shimokasuya, Isehara, Kanagawa, 259-1193, Japan

${ }^{4}$ Division of Organ Replacement Research, Center for Molecular Medicine, Jichi Medical University, 3311-1 Yakushiji, Shimotsuke, Tochigi, 329-0498 Japan

\begin{abstract}
Although intervertebral disc herniation and associated sciatica is a common disease, its molecular pathogenesis is not well understood. Immune responses are thought to be involved. This study provides direct evidence that even nondegenerated nucleus pulposus (NP) cells elicit immune responses. An in vitro colony forming inhibition assay demonstrated the suppressive effects of autologous spleen cells on NP cells and an in vitro cytotoxicity assay showed the positive cytotoxic effects of natural killer (NK) cells and macrophages on NP cells. Non-degenerated rat NP tissues transplanted into wild type rats and immunedeficient mice demonstrated a significantly higher NP cell survival rate in immune-deficient mice. Immunohistochemical staining showed the presence of macrophages and NK cells in the transplanted NP tissues. These results suggest that even non-degenerated autologous NP cells are recognized by macrophages and NK cells, which may have an immunological function in the early phase of disc herniation. These findings contribute to understanding resorption and the inflammatory reaction to disc herniation.
\end{abstract}

Keywords: Nucleus pulposus, immune response, macrophage, natural killer cell, intervertebral disc, autoimmunity.

*Address for correspondence:

Kunihiko Murai

Department of Anesthesiology and Intensive Care Medicine,

Jichi Medical University,

3311-1 Yakushiji, Shimotsuke, Tochigi, 329-0498, Japan

Telephone Number: +81 285587383

FAX Number: +81285444108

E-mail: murai.mane@jichi.ac.jp

\section{Introduction}

Resorption of herniated nucleus pulposus (NP) is a clinically demonstrated phenomenon during intervertebral disc herniation. In understanding the undefined pathogenesis of intervertbral disc herniation and sciatica, clarifying the molecular events that occur in resorption of NP is important. Nachemson (1969) reported decreased $\mathrm{pH}$ levels within and around a herniated lumbar disc and speculated that sciatica was caused by an inflammatory reaction surrounding the nerve root. Subsequently, various inflammatory chemical factors secreted from herniated NP, including tumor necrosis factor (TNF)- $\alpha$ (Weiler et al., 2005; Le Maitre et al., 2007), interleukin (IL)-1 $\beta$ (Le Maitre et al., 2007) and nitric oxide (NO) (Katsuno et al., 2008), have been implicated as causes of sciatica (McCarron et al, 1987; Geiss et al., 2007). Further, the production of matrix metalloproteinases (MMPs) has been implicated in the resorption of the herniated NP (Doita et al., 2001).

Bobechko and Hirsh (1965) and Gertzbein et al. (1975) reported that herniated NP tissue is recognized as a foreign antigen that induces an autoimmune response producing inflammation. Later, immunohistochemical (IHC) analyses of human herniated discs revealed the presence of infiltrated T cells (Park et al., 2001), macrophages (Park et al., 2001; Virri et al., 2001), and antigen-antibody complexes in the NP (Satoh et al., 1999). An in vitro co-culture model of macrophages and NP cells also showed the infiltration of macrophages and a decreased wet weight of the NP (Haro et al., 2000). The expression of IL-6, -8, -12, and interferon (IFN)- $\gamma$ suggests Th1 lymphocyte activation (Kang et al., 1996; Burke et al., 2002; Park et al., 2002). Geiss et al. placed autologous porcine NP in subcutaneous titanium chambers and observed the infiltration of activated $\mathrm{T}$ and $\mathrm{B}$ cells (Geiss et al., 2007), including IL-4-producing Th2 cells and $\gamma \delta$ $\mathrm{T}$ cells (Geiss et al., 2008). These results indicate both innate and acquired immune responses to the NP. Other studies (Park et al., 2001; Jones et al., 2008), however, have reported that NP cells undergo apoptosis and are phagocytised by macrophages without an immune response. Ikeda et al. (1996) investigated infiltrated cells consisting of macrophages and a small number of $T$ cells, and proposed that extruded or sequestrated disc material 
has the potential to be absorbed by phagocytes. It remains unclear from these reports whether immune responses are truly involved in disc herniation, and if so, which immune cells initiate the immune response.

In order to investigate whether an immune response is involved in disc herniation, fundamental research on NP cells and the immune system is required. The purpose of this study is to clarify the immune response to autologous NP cells and to identify the specific immune cells that initiate an immune response by using in vivo and in vitro rat models to assess the survival of NP cells exposed to immune system cells.

\section{Materials and Methods}

\section{In vitro studies}

Preparation of rat-tail NP cells. Male Sprague-Dawley (SD) rats (Nihon Charles River Co., Kanagawa, Japan) aged 10-12 weeks, were used for the colony forming inhibition assay (CFI), and male Lewis rats (Nihon Charles River) aged 10-12 weeks were used for the cytotoxicity assay. Following sacrifice, NP tissues were dissected from the whole tail and digested in $0.05 \%$ trypsin-ethylene diamine tetraacetic acid (EDTA; Gibco, Grand Island, NY, USA) for 15 minutes. The digestate was washed, passed through a $100 \mu \mathrm{m}$ mesh cell strainer, the NP cells were collected by mild centrifugation (500Gx4min). These experiments were approved by the Animal Research Committee of Tokai University (071095) and conducted according to the guidelines for animal experiments.

Preparation of spleen cells. Autologous spleen cells were used as effector cells for the CFI assay and isogenous spleen cells were used for the cytotoxicity assay. Briefly, the spleens were removed, mashed and passed through a $100 \mu \mathrm{m}$ mesh cell strainer. Red blood cells were haemolysed using $0.8 \% \mathrm{NH}_{3} \mathrm{Cl}$. The spleen cells were collected by mild centrifugation ( $500 \mathrm{Gx} 4 \mathrm{~min})$. The spleen cells $\left(10^{7}\right.$ cells $\left./ \mathrm{ml}\right)$ were then incubated in RPMI-1640 medium (Invitrogen, Grand Island, NY, USA) with 15\% foetal bovine serum (FBS, Qualified FBS, Invitrogen) at $37^{\circ} \mathrm{C}$ for five hours with IL-2 $(60 \mathrm{IU} / \mathrm{ml}$; Imunase, Shionogi, Osaka, Japan ).

Purification of T cells, natural killer (NK) cells and macrophages. For cytotoxicity assays, more than $10^{8}$ of the spleen cells isolated from a Lewis rat were suspended in fluorescence-activated cell sorting (FACS) buffer (Facs Flow, Becton Dickinson (BD) Pharmingen, Tokyo, Japan) and incubated for 30 minutes at $4^{\circ} \mathrm{C}$ with saturating amounts of the following antibodies: CD3 (\#550353, PE mouse anti-rat CD3, BD), CD4 (\#550057 Pharmingen, APC mouse anti-rat CD4, BD), CD8 (\#558824, Per CP mouse anti-rat CD8a, BD), CD161 (\#550978, biotin mouse anti-rat CD161, BD). The labelled spleen cells were then separated into NK cells (CD161+), CD4+T cells $(\mathrm{CD} 3+\mathrm{CD} 4+), \mathrm{CD} 8+\mathrm{T}$ cells $(\mathrm{CD} 3+\mathrm{CD} 8+)$, and macrophages (remaining CD3-) using a FACS Vantage (BD).
CFI assay. For the CFI assay, the suppressive effect of immune cells (effector cells) on colony formation by autologous NP cells (target cells) was assessed by a previously described method (Spitzer et al., 1980). The NP cells isolated from $\mathrm{SD}$ rats $(\mathrm{N}=4)$ were immediately utilized for the assay procedures. NP cells $\left(6 \times 10^{3}\right)$ and autologous spleen cells were seeded for each $\mathrm{E}: \mathrm{T}$ ratio of $0: 1,25: 1,50: 1$ and 100:1 in $6 \mathrm{ml}$ of $0.9 \%$ methylcellulose formation (MethoCult H4230 Stemcell Technologies, Vancouver, Canada) in a single tube, mixed completely, then we dispensed it by $1 \mathrm{ml}$ in $35 \mathrm{~mm}$ dishes $(\mathrm{n}=4$ for each $\mathrm{E}: \mathrm{T}$ ratio). The dishes were incubated at $37^{\circ} \mathrm{C}$ in $5 \% \mathrm{CO}_{2}$ and full humidity for 14 days without medium replacement, after which the number of NP colonies was scored at least twice for each dish using a tally board on the bottom of the dishes.

Cytotoxicity assay. For the cytotoxicity assay, NP cells from Lewis rats $(\mathrm{N}=2)$ were monolayer cultured in RPMI1640 medium with $15 \%$ FBS for 10 days. The cells were labeled using calcein-AM (Dojin Chemical Institute, Kumamoto, Japan) for 60 minutes at $37^{\circ} \mathrm{C}$ without serum, washed, and seeded into 96-well V-bottomed plates (\#4914, Matrix Technologies, Hudson, NH, USA) at 1×104 cells/ well. Suspensions of purified isogenous NK cells, CD4+ T cells, CD8+ T cells, or macrophage cells were then added to wells at $E: T$ cell ratios of $0: 1,25: 1,50: 1$ and 100:1 in a final volume of $200 \mu \mathrm{L} /$ well in RPMI-1640 medium without serum ( $\mathrm{n}=4$ for each ratio). The plate was centrifuged, then incubated in humidified air for eight hours at $37^{\circ} \mathrm{C}$. After incubation, the plates were centrifuged and $100 \mathrm{~mL}$ of supernatant from each well was moved to another 96 well flat-bottomed plate in the same pattern, and was measured using a fluorescent plate reader $\left(\lambda_{\text {ex }}=485\right.$ $\mathrm{nm}, \lambda_{\mathrm{em}}=520 \mathrm{~nm}$, Beckman Coulter, Brea, CA, USA). Cytotoxic activity was determined according to a modification of the ${ }^{3} \mathrm{H}$-uridine labelling method described by Wang et al. (1993). Cytotoxicity was calculated as:

$\%$ cytotoxicity $=\frac{\text { experimental release }- \text { spontaneous release }}{\text { total release }- \text { spontaneous release }} \times 100(1)$

Total release was obtained by detergent solubilisation in the presence of 1\% Triton X-100 (GE Healthcare Japan, Tokyo, Japan). Spontaneous release means the fluorescence release of the pure NP cell groups. Fibroblastic cells from the anulus fibrosus was also analyzed as negative control.

\section{In vivo study}

For in vivo studies, intact rat NP tissues were transplanted with PBS into immunodeficient mice and wild type rats. The survival rate of the NP cells in the transplanted tissue was measured using the bioluminescence imaging (BLI) method described below to estimate the influence of immunity on NP cell survival. IHC staining was done on the NP tissues from the rat model to detect attracted immune cells, which would indicate the initiation of an immune response.

\section{Transplantation of NP for the BLI study}

For the BLI analysis, four male Lewis rats 10-12 weeks of 
age were used as recipients of NP tissues for the Lewis to Lewis (Lew-Lew) group and four male 10-12-week-old NOD/Shi-scid mice (Nihon Charles River) served as recipients of NP tissues for the Lewis to NOD (Lew-NOD) group. Transgenic (Tg) male Lewis rats (8-10 weeks of age) whose tissues express luciferase produced by repeated crossing of $\mathrm{Tg}$ rats and confirmed in the Organ Replacement Research Department in Jichi Medical University were used as NP tissue donors. $100 \mu \mathrm{g}$ of NP tissues were injected with $100 \mu \mathrm{L}$ of PBS under the abdominal skin of recipients under general anaesthesia using 2-3\% isoflurane. One donor was used for each recipient. The BLI study was conducted using a IVIS system (Xenogen Corp., Hopkinton, MA, USA) with LivingImaging acquisition and analysis software. Briefly, animals were anesthetized with isoflurane and given 125 $\mathrm{mg} / \mathrm{kg}$ D-luciferin substrate (Biosynth AG, Staad, Switzerland). The animals were then placed in a light-tight chamber for imaging with a CCD camera. The photon counts from the peak luciferase activity were recorded. Luciferase activity was measured as photons emitted/ second. Imaging studies were performed immediately after transplantation and at day 7 , day 14 and day 21 .

\section{IHC staining}

For IHC staining, male Lewis rats $(n=6)$ were newly used as recipients of NP tissues. The transplantation procedure was the same as for the Lew-Lew group described above and one donor was used for each recipient $(n=6)$. Two recipients were sacrificed at 5,10, and 40 days after transplantation. In addition, two NOD mouse in BLI study was sacrificed at 26 days after transplantation. After fixation with $10 \%$ formalin for three days, a paraffin block was made though an alcohol-xylene-paraffin graded series. Five-micron thick paraffin sections were cut sagittally from the epidermis to the peritoneal membrane across the transplantation site, deparaffinized 5 - $\mu \mathrm{m}$ sections first were rehydrated through xylene and graded alcohol series. For double-staining immunofluorescence, tissue slides were incubated overnight at $4^{\circ} \mathrm{C}$ with a primary monoclonal antibody to keratan sulphate (KS) (\#270427-1, mouse antiKS, Associates of Cape Cod, Falmouth, MA, USA), diluted $1: 100$ in PBS with $1 \%$ BSA, followed by incubation in darkness at room temperature for three hours with Alexa Fluor 488-conjugated anti-mouse IgG diluted 1:200. After washing with PBS, the slides were incubated overnight at $4^{\circ} \mathrm{C}$ in darkness with diluted $(1: 100)$ primary antibodies for rat $\mathrm{T}$ cells (\#550353, PE mouse anti-rat CD3, BD), macrophages (\#sc-9139, rabbit anti-rat CD68, Santa Cruz Biotechnology, CA, USA), or NK cells (\#550978, Biotin mouse anti-rat CD161, BD). After washing with PBS, the slides with CD68 were incubated for 60 minutes in room temperature with anti-rabbit goat Alexa 594 antibody (Invitrogen); slides with CD161 staining were incubated for one hour with streptavidin-Alexa 594 (Invitrogen). All slides were then covered with Vectashield mounting medium with DAPI (H-1500, Vector Laboratories, Burlingame, CA, USA). Sample sections of day 5, day 10 and day 40 were also stained with HE and Safranin-O.

\section{Data Analysis}

All data are given as the mean \pm standard deviation (SD). The statistics were processed by Excel Stat 2006 (SSRI, Tokyo, Japan). Two-factor analysis of variance (ANOVA) was employed to analyze the in vitro and in vivo results. The Mann-Whitney U-test was used to compare the results of the two groups in CFI assay. When significant differences were revealed by the ANOVA, post hoc comparisons were done. Statistical significance was defined as $p<0.05$.

\section{Results}

\section{In vitro study}

CFI assay. Spleen cells from SD rats were used as effector cells for autologous tail NP target cells. Colony formation assays showed two types of colonies that were identified as CFU-A (adherent) and CFU-NA (non-adherent) when counting colonies. Without effector cells (E:T cell ratio of $0: 1)$, NP cells $\left(1 \times 10^{3}\right)$ formed CFU-NA colonies ranging in numbers from 82-118 (94.8 \pm 18.1$)$ and CFU-A colonies ranging from 39-60 (48.3 \pm 9.6$)$. When effector cells were added, NP cells $\left(1 \times 10^{3}\right)$ with an E:T cell ratio of $25: 1$ yielded CFU-NA colonies ranging from 26-31 (29.0 \pm 2.2$)$ and CFU-A colonies ranging from $22-38$ (28.3 \pm 6.8$)$, an E:T cell ratio of 50:1 resulted in CFU-NA colonies ranging from 19-26 (22.8 \pm 3.0$)$ and CFU-A colonies ranging from 19-34 (26.8 \pm 6.6$)$ and an E:T cell ratio of 100:1 produced CFU-NA colonies ranging from 19-25 (21.0 \pm 2.8$)$ and CFU-A colonies ranging from 18-27 (21.0 44.2$)$. The suppressive effect of spleen cells was apparent (Fig. 1A). CFU-NA colonies were affected stronger than CFU-A colonies (Table 1). Microscopic examinations of CFU-NA

Table 1 Percentage of the number of colonies to the control $(E: T$ cell ratio $=0: 1)$

\begin{tabular}{lcccc} 
E:T cell ratio & $0: 1$ & $25: 1$ & $50: 1$ & $100: 1$ \\
\hline CFU-NA (\%) & 100 & $30.6 \pm 2.3$ & $24.0 \pm 3.2$ & $22.2 \pm 3.0$ \\
CFU-A (\%) & 100 & $58.5 \pm 14.2$ & $55.4 \pm 13.7$ & $43.5 \pm 8.8$ \\
\hline$p$-value & & $p=0.021$ & $p=0.021$ & $p=0.019$ \\
\hline
\end{tabular}




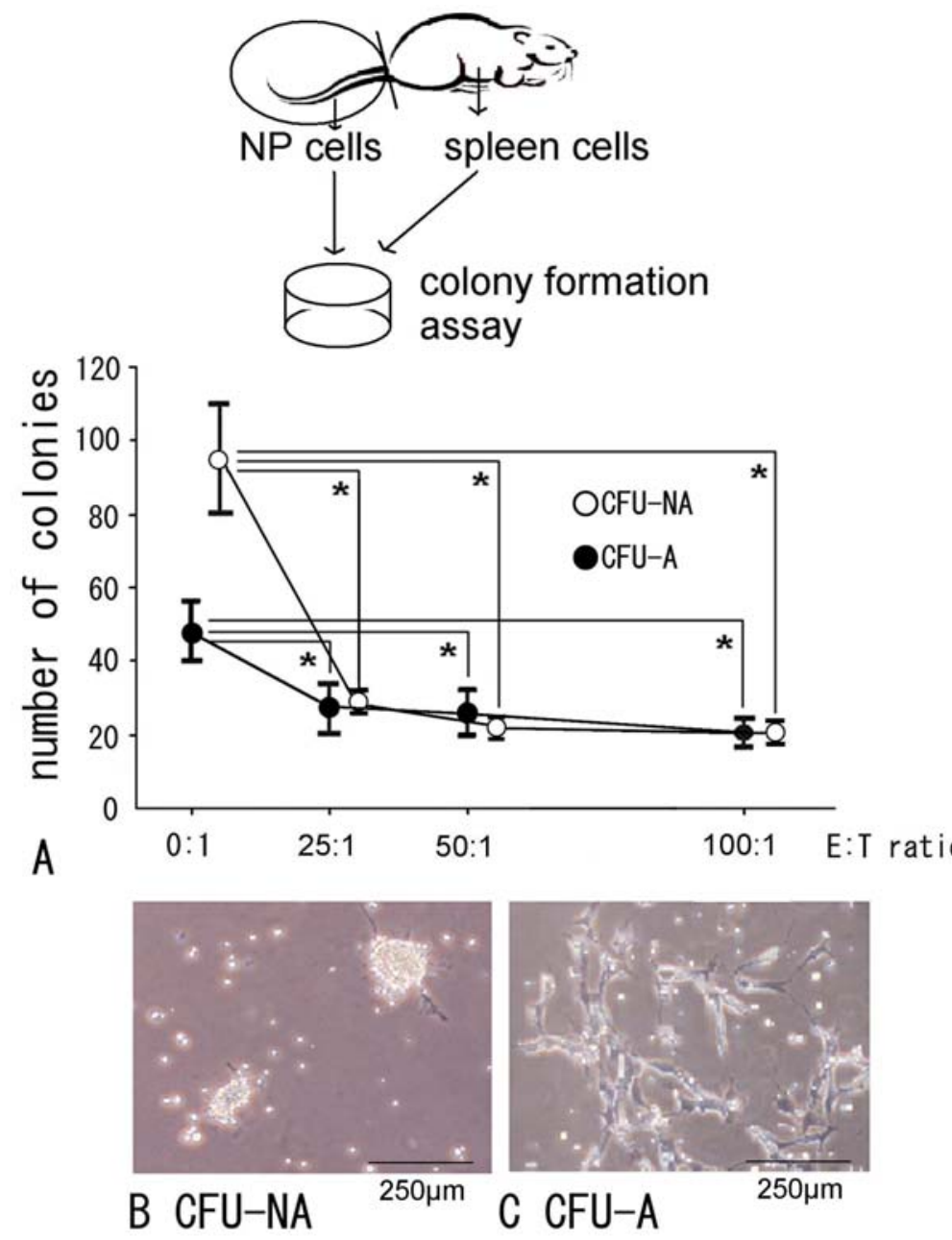

Fig. 1. (A) Results of CFI assay in vitro. Numbers of CFU-NA (open circle) and CFUA (closed circle) colonies in the $\mathrm{E}$ : $\mathrm{T}$ ratio of $0: 1,25: 1,50: 1$ and 100:1 were counted at day 14. Colony formation of NP cells was suppressed by the addition of autologous spleen cells in both groups $(* p<0.05$ compared with that in the E:T ratio of $0: 1$ ). (B) Attraction of spleen cells to CFU-NA. (C) Attraction of spleen cells to CFU-A. The larger number of spleen cells attracted to CFU-NA than to CFU-A supports the result in our current study that CFU-NA is more sensitive to autologous spleen cells.

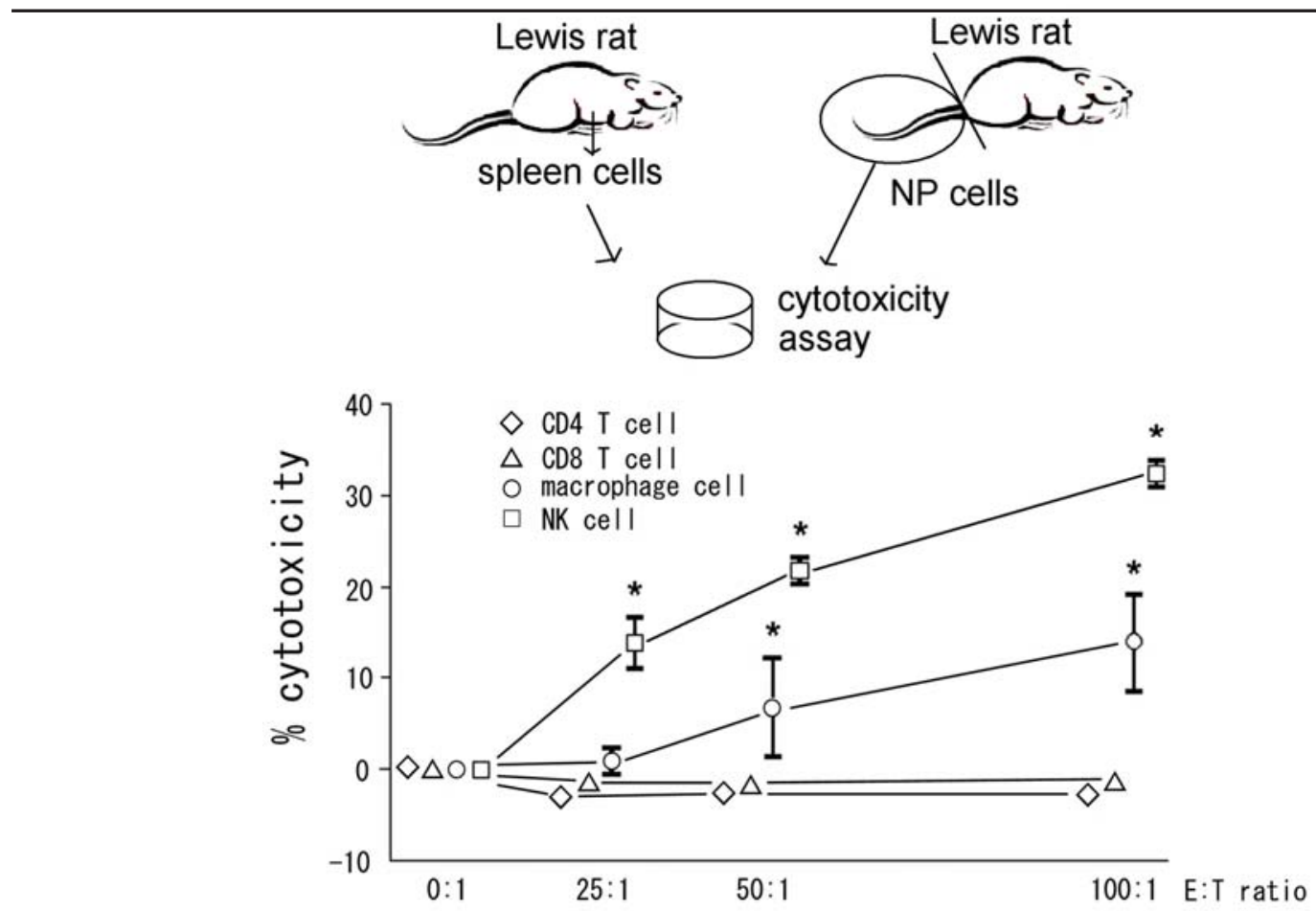

Fig. 2. Results of cytotoxicity assay in vitro. Cytotoxicity was calculated as follows,

$$
\% \text { cytotoxicity }=\frac{\text { experimental release }- \text { spontaneous release }}{\text { total release }- \text { spontaneous release }} \times 100
$$

$0 \%=$ no cytotoxicity, $100 \%=$ maximum cytotoxicity as strong as detergent agent.

Cytotoxicity caused by NK cells and macrophages was suggested as a result of 8 hrs coculture $(* p<0.05$ compared with that in the E:T ratio of $0: 1$ ). 


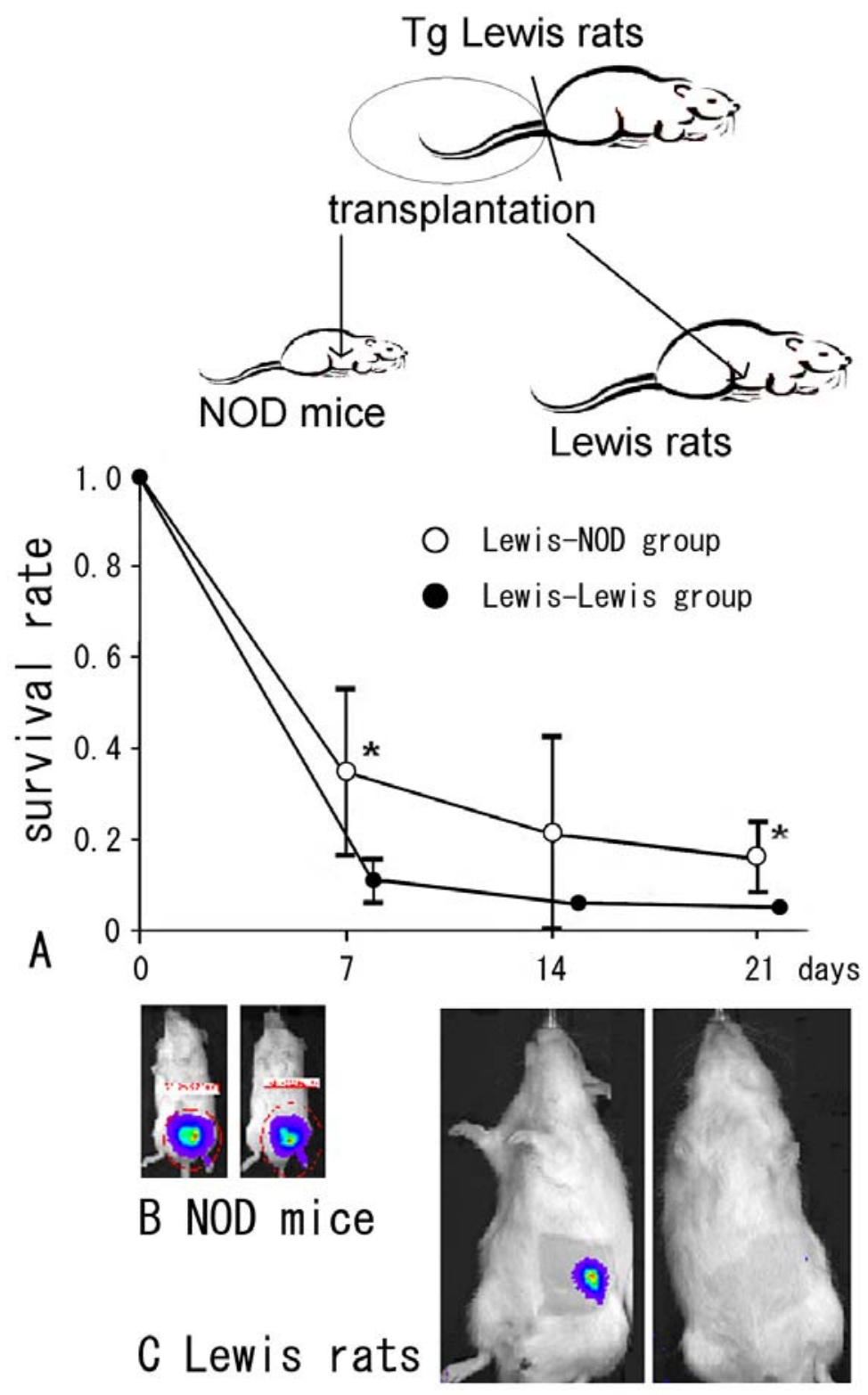

Fig. 3. (A) Survival rate of transplanted NP cells in Lewis rats and NOD mice ( $n=4$, each). Closed circle indicates Lewis-Lewis group and open circle indicates Lewis-NOD group. Because intensity of luminescence is positively linear to the number of NP cells (data not shown), survival rate of NP cells was calculated as follows:

$$
\text { Survival rate }=\frac{\text { Intensity of luminance after } 7,14 \text { or } 21 \text { days }}{\text { Intensity of luminance just after transplantation }}
$$

so that baseline value of survival rate (day 0) is " 1 ". The survival rate was higher in the Lewis-NOD group than in the Lewis-Lewis group $(* p<0.05)$. (B) BLI imaging of NOD mouse at day 0 (left) and at day 90 (right). (C) BLI imaging of Lewis rat at day 0 (left) and at day 21 (right). NP cells hardly survived at day 21 .

(Fig. 1B) and CFU-A (Fig. 1C) colonies revealed that larger numbers of spleen cells were attracted to CFU-NA colonies than to CFU-A colonies, further indicating that CFU-NA colony formation was more sensitive to the presence of spleen cells.

Cytotoxicity assay. From $10^{8}$ Lewis rat spleen cells, $3.0 \times 10^{7} \mathrm{CD} 4+\mathrm{T}$ cells, $2.0 \times 10^{7} \mathrm{CD} 8+\mathrm{T}$ cells, $1.0 \times 10^{7}$ macrophages and $6.0 \times 10^{6} \mathrm{NK}$ cells were sorted by FACS with data showing that more than $95 \%$ of the cells were alive.
Cytotoxicity to autologous NP cells was proportional to the E:T cell ratio in NK cells and macrophages (Fig. 2). At an E:T cell ratio of 100:1, cytotoxicity was $31-35 \%$ in NK cells and $9-20 \%$ in macrophages. Significant cytotoxicity was observed in NK cells at E:T cell ratios of $25: 1$ or more $(p<0.0001)$ and in macrophages at E:T cell ratios of 50:1 or more compared to the corresponding values in the absence of effector cells ( $\mathrm{p}=0.001$ at $50: 1 ; p$ $<0.0001$ at 100:1) (Fig. 2). CD4+T cells and CD8+T cells did not have cytotoxic effects on NP cells. 

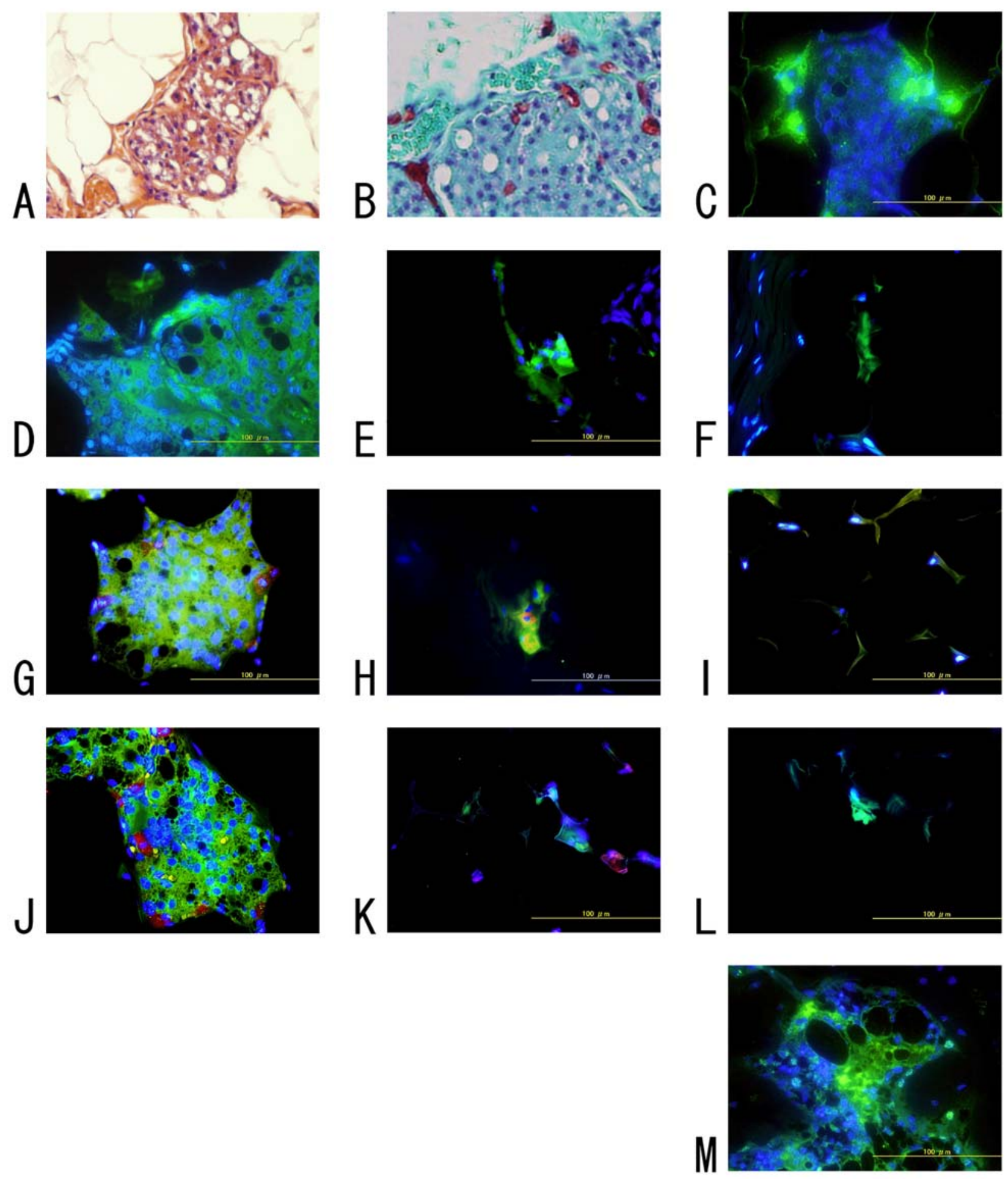

Fig. 4. Histological analysis of NPs at the transplant site of recipient rats. HE (A), safranin-O (B) and keratan sulphate IHC (C) staining indicate the presence of transplanted NPs at day 5. Safranin-O stains the proteoglycan of NPs (Red), and keratan sulphate is specific extracellular matrix of NPs (Green). Keratan sulphate was stained even 26 days after transplantation in the recipient of NOD mouse $(\mathrm{M})$.

CD3 (D F), CD68 (G I) and CD161 (J L) (Red) are immunohistochemically double-stained with keratin sulphate (Green). D, G, and J are the results at day 5; E, H, and K at day 10; F, I, and L at day 40. Keratan sulphate decreased dependent on time, NK cells and macrophages decreased simultaneously. 
To summarize, the results of the in vitro CFI and cytotoxicity assays revealed the presence of a spleen cell population that had cytotoxic effects on autologous NP cells. This cytotoxic spleen cell population is composed of sub-populations of NK cells and macrophages. Furthermore, as a negative control of cytotoxicity assay, we used fibroblast-like cells of annulus fibrosus origin. The result was that the fibroblast-like cells was tolerant to isogeneous spleen cells, however nucleus pulposus cells was sensitive. This result raised our test hypothesis that NP is sensitive to specific immune cells.

\section{In vivo study}

BLI study. We performed a BLI study to investigate immunological responses to transplanted NP tissues in vivo. The BLI evaluation showed a significantly higher survival rate for transplanted NP cells in the Lew-NOD group compared to that in the Lew-Lew group totally $(p=0.036)$, at day $7(0.35 \pm 0.18 v s .0 .11 \pm 0.05 ; p=0.042)$ and at day $21(0.16 \pm 0.08$ vs. $0.03 \pm 0.02 ; p=0.037)$ (Fig. 3A). After 90 days, up to $13 \%$ of the transplanted NP cells had survived in the Lew-NOD group (Fig.3B). NP cells transplanted into Lewis rats (Lew-Lew) did not survive past 21 days, when luminescence at the NP cell transplant site had decreased to near background levels (Fig.3C).

IHC staining. Because our results showed that transplanted NP cell survival was reduced in association with an immunological reaction, we used immunological staining to identify which types of immune cells had infiltrated. Transplanted NP tissues in rats at day 5 existed mainly in the loose subcutaneous fat tissue as an agglomeration of cells with a bubble-like extracellular matrix (Fig. 4A). Safranin-O staining showed the presence of red-stained proteoglycans (Fig. 4B), and fluorescent green-stained keratan sulphate, both of which are constituents of the extracellular matrix of the NP (Fig. 4C). We observed transplanted NP tissues at day 5 (Fig. 4D, G,
J), however, the amount of transplanted NP tissue was markedly decreased at day 10 in the Lewis rats, while NP tissue was obviously present in NOD mice recipients even at day 26 (Fig. 4M). From the IHC evaluation of immune cells in Lewis rats, no $\mathrm{CD} 3$ positive T cells attraction were observed subcutaneously from day 5 to day 40 (Fig. 4D, $\mathrm{E}, \mathrm{F})$. Attraction of NK cells and macrophages was observed at days 5 and 10 around the outgrown NP tissues (Fig. 4G, H, J, K); however, neither NP tissues nor immune cells were observed at day 40 (Fig. 4I, L). The numbers of NK cells and macrophages in microscope fields that included agglomerated NP cell clusters decreased with time (Table 2).

\section{Discussion}

The precise mechanism of immunological involvement in the pathology of disc herniation has not been defined. We performed two immunological assays, the CFI and the cytotoxicity assay, using co-cultured NP and immune cells. We also developed an in vivo subcutaneous transplantation model and measured the survival rate of transplanted NP cells using the BLI method. IHC at the transplant site of the recipient rats was used to identify the immune cells.

The suppression of NP cell colony formation was observed to be dependent on the effector:target (E:T) cell ratio. We found that non-adherent (CFU-NA) colonies were more strongly suppressed by immune cells than adherent (CFU-A) colonies. Because NP cells are known to be heterogeneous (Chelberg et al., 1995), this difference in colony formation may reflect the different epitopes recognized by immune cells, or possible differences in the immune privilege function, like the presence or absence of Fas ligand. Based on these possibilities, about $20 \%$ of the NP cell population were alive even at E:T cell ratio of 100:1, which appears to differ immunologically from other NP cells. Of particular interest was the assessment of direct

Table 2 The number of immune cells in a microscope field (x40)

Day $5 \quad$ Day $10 \quad$ Day 40

\begin{tabular}{lccc}
\hline T cell & None & None & None \\
NK cell & $2-5$ & 1 & None \\
Macrophage & $4-7$ & $1-3$ & None \\
\hline
\end{tabular}

This table indicates the number of representative agglomerated NP cell clusters in the tissue specimen of transplanted site in Lewis rats. NK and macrophage cells were observed in the transplanted site in the early phase, whereas T cells were not observed. In day 40, neither NP tissues nor immune cells were observed. 
cytotoxic function of the immunological cell types. The results of the cytotoxicity assays of isolated T, NK, and macrophage cells demonstrated that only the NK and macrophage cells had cytotoxic activity on NP cells. The target molecules and their location on the NP cells remain undefined; further biological and immunological studies are necessary.

The results of the BLI study showed differences in the survival rate of NP cells in the transplanted NP tissues between the Lewis rat and NOD mouse recipients. NP cells are known to undergo apoptosis (Park et al., 2001), and intervertebral disc cells are thought to be able to behave as competent phagocytes (Jones et al., 2008). However, these results do not explain the different survival rates of NP cells in the current study. Because the survival rate of NP cells was higher in immunodeficient mice than in Lewis rats, immunological functions are implicated. NOD/shiscid mice lack mature lymphocytes, and have macrophage dysfunction, a reduced level of NK cell activity and absence of circulating immune components compared to wild-type mice. These factors may account for the difference in NP cell survival rate between NOD mice and Lewis rats in our study.

We also detected the infiltration of specific immune cells into the NP transplant sites; these results definitively demonstrate the immunological activity of these cell types against NP tissues. Macrophages and NK cells, but not T cells, were detected, although the presence of T and B cells in isolated human herniated discs and in experimental porcine models has been previously reported (Geiss et al., 2007). Our results suggest an early immunological response after normal NP tissues were exposed to the immune system. Thus, macrophages and NK cells were observed on days 5 and 10 when residual NP was present, but not on day 40 when the transplanted NP had disappeared. This finding supports the presence of an immunological response to transplanted NP tissues.

In our IHC study, CD68 positive cells did not resemble the appearance of resident chondrocytes. Although Jones et al. (2008) suggested that CD68 positive cells were transformed resident intervertebral disc cells, based on their morphology, the results of our study show that these macrophages are not transformed resident cells but rather are infiltrating cells.

Autologous tissues are generally not recognized as foreign by the immune system. The NP is an immuneprivileged tissue isolated from the immune system (Hiyama et al., 2008) and it, like similarly isolated tissues, including the eye and testis, can produce inflammatory autoimmune responses (Wildner and Diedrichs-Möhring, 2004; Schuppe and Meinhardt, 2005). Another possible trigger for autoimmunity is innate immunity, which is induced by chemical factors without specific antigen-antibody responses, leading to rapid immune responses to pathological microbe antigens. Because the NP cell produces chemical factors and the carbohydrate structure of the extracellular matrix produced by NP cells may mimic that of pathological microbe antigens, the NP may trigger an innate immunity response (Bárdos et al., 2005).

In the in vivo transplantation model, we utilized a xenogeneic model because the mouse is too small to obtain enough donor NP cells. The use of NOD mice as recipients is well established for evaluating the effects of immunodeficiency. In addition, the xenogeneic transplantation model is commonly used for immunological evaluation (Yoshino et al., 2000).

In conclusion, even non-degenerated NP cells elicit an immune response, and macrophages and NK cells in particular are shown to have an early immunological function when NP cells are exposed to the immune system. While these results may not be directly applicable to the human, this study provides important information for understanding the pathophysiological mechanism of disc herniation.

\section{Acknowledgements}

This work was supported in part by a Grant-in-Aid for Scientific Research and a Grant of The Science Frontier Program from the Ministry of Education, Culture, Sports, Science and Technology of Japan (D.S. and J.M.), grants from AO Spine International (D.S.), and Jichi Medical University.

\section{References}

Bárdos T, Szabó Z, Czipri M, Vermes C, TunyogiCsapó M Urban MR, Mikecz K, Glant TT (2005) A longitudinal study on an autoimmune murine model of ankylosing spondylitis. Ann Rheum Dis 64: 981-987.

Bobechko WP, Hirsh C (1965) Auto-immune response to nucleus pulposus in the rabbit. J Bone Joint Surg Br 47: 574-580.

Burke JG, Watson RWG, McCormack D, Dowling FE, Walsh MG, Fitzpatrick JM (2002) Spontaneous Production of Monocyte Chemoattractant Protein-1 and Interleukin8 by the Human Lumbar Intervertebral Disc. Spine 27: 1402-1407.

Chelberg MK, Banks GM, Geiger DF, Oegema TR Jr (1995) Identification of heterogeneous cell populations in normal human intervertebral disc. J Anat 186: 43-53.

Doita M, Kanatani T, Ozaki T, Matsui N, Kurosaki M, Yoshiya S (2001) Influence of macrophage infiltration of herniated disc tissue on the production of matrix metalloproteinases leading to disc resorption. Spine 26: 1522-1527.

Geiss A, Larsson K, Rydevik B, Takahashi I, Olmarker K (2007) Autoimmune Properties of Nucleus Pulposus: An Experimental Study in Pigs. Spine 32: 168-173.

Geiss A, Larson K, Junevik K, Rydevik B, Olmarker K (2008) Autologous nucleus pulposus primes T cell to develop into interleukin-4-producing cells: an experimental study on the autoimmune properties of nucleus pulposus. J Orthop Res 27: 97-103.

Gertzbein M, Tail M, Gross A, Falk R (1975) Autoimmunity in degenerative disc disease of the lumbar spine. Orthop Clin North Am 6: 67-73.

Haro H, Craford HC, Fingleton B (2000) Matrix metalloproteinase-7-dependent release of tumor necrosis 
factor- $\alpha$ in a model of herniated disc resorption. J Clin. Invest., 105: 143-150.

Hiyama A, Mochida J, Iwashina T, Omi H, Watanabe T, Serigano K, Tamura F, Sakai D (2008) Transplantation of mesenchymal stem cells in a canine disc degeneration model. J Orthop Res 26: 589-600.

Ikeda T, Nakamura T, Kikuchi T, Umeda S, Senda H, Takagi K (1996) Pathomechanism of spontaneous regression of the herniated lumbar disc: Histologic and immunohistochemical study. J Spinal Disord 9: 136-140.

Jones P, Gardner L, Menaga J, Williams G, Roberts S (2008) Intervertebral disc cells as competent phagocytes in vitro: implications for cell death in disc degeneration. Arthritis Res Ther 10: R86.

Kang JD, Georgescu HI, McIntyle-Larkin L, Stefanovic-Racic M, Donaldson WF 3rd, Evans CH (1996) Herniated lumbar intervertebral discs spontaneously produce matrix metalloproteinases, nitric oxide, interleukin-6, prostaglandin E2. Spine 21: 271-277.

Katsuno R, Hasegawa T, Iwashina T, Sakai D, Mikawa Y, Mochida J (2008) Age-related effects of cocultured rat nucleus pulposus cells and macrophages on nitric oxide production and cytokine imbalance. Spine 33: 845-849.

Le Maitre CL, Hoyland JA, Freemont AJ (2007) Catabolic cytokine expression in degenerate and herniated human intervertebral discs: IL-1b and TNFa expression profile. Arthritis Res Ther 9: R77.

McCarron RF, Wimpee MW, Hudkins PG, Laros GS (1987) The inflammatory effect of nucleus pulposus. A possible element in the pathogenesis of low back pain. Spine 12: 760-764.

Nachemson A (1969) Intradiscal measurements of $\mathrm{pH}$ in patients with lumbar rhizopathies. Acta Orthop Scand 40: $23-42$.

Park JB, Chang H, Kim KW (2001) Expression of Fas ligand and apoptosis of disc cells in herniated lumbar disc tissue. Spine 26: 618-621.
Park JB, Chang H, Kim YS (2002) The pattern of interleukin-12 and T-helper types 1 and 2 cytokine expression in herniated lumbar disc tissue. Spine 27: 21252128.

Satoh K, Konno S, Nishiyama K, Olmarker K, Kikuchi S (1999) Presence and distribution of antigen-antibody complexes in the herniated nucleus pulposus. Spine 24: 1980-1984.

Schuppe HC, Meinhardt A (2005) Immune privilege and inflammation of the testis. Chem Immunol Allergy 88: 1-14.

Spitzer G, Verma DS, Fisher R, Zander A, Vellekoop L, Litam J (1980) The myeloid progenitor cell - its value in predicting hematopoietic recovery after autologous bone marrow transplantation. Blood 55: 317-323.

Virri J, Gronblad M, Seitsalo S, Habetemarian A, Kappa E, Karahaju E (2001) Comparison of the prevalence of inflammatory cells in subtypes of disc herniations and associations with straight leg raising. Spine 26: 2311-2315.

Wang XM, Terasaki PI, Rankin GW Jr., Chia D, Zhong HP, Hardy S (1993) A new microcellular cytotoxicity test based on calcein AM release. Hum Immunol 37: 264-270.

Weiler C, Nerlich BE, Boos N (2005) Expression and distribution of tumor necrosis factor alpha in human lumbar intervertebral discs: A study in surgical specimen and autopsy controls. Spine 30: 44-53.

Wildner G, Diedrichs-Möhring M (2004) Autoimmune uveitis and antigenic mimicry of environmental antigens. Autoimmun Rev 3: 383-387.

Yoshino H, Ueda T, Kawahata M, Kobayashi K, Ebihara Y, Manabe A, Tanaka R, Ito M, Asano S, Nakahata T, Tsuji K (2000) Natural killer cell depletion by anti-asialo GM1 antiserum treatment enhances human hematopoietic stem cell engraftment in NOD/Shi-scid mice. Bone Marrow Transplantation 26: 1211-1216. 